\title{
Niosomes and liposomes as promising carriers for dermal delivery of Annona squamosa extract
}

\author{
Ebtesam Abdelghany Mohamad ${ }^{\circledR 1 *}$, Heba Mohamed Fahmy ${ }^{1}$ \\ ${ }^{1}$ Biophysics Department, Faculty of Science, Cairo University, Giza, Egypt
}

\begin{abstract}
The leaf extract of Annona squamosa L. has antibacterial, antidiabetic, antioxidant, and anticancerous activities. The present work aims to compare between liposomes and niosomes as carriers for A. squamosa extract to improve its transdermal bioavailability. Physical characterization for niosomes and liposomes was performed using: transmission electron microscope (TEM), scanning electron microscope (SEM) and Fourier transform infrared spectroscopy (FTIR). In addition, the encapsulation efficiency for $A$. squamosa in both carriers was evaluated and in-vitro drug release experiments were performed. The results proved the potential of both carriers to penetrate the outer layer of the skin (stratum corneum) which is considered as a strong barrier against the diffusion of many compounds through the skin. Moreover, the results pointed out that niosomes and liposomes lasted long time through the skin, which ensures the presence of antioxidant extract in the skin for prolonged periods. This would have a benefit of targeting free radicals in the skin. The encapsulation efficiency of liposomes for $A$. squamosa extract exceeded that of niosomes, however, niosomes demonstrated longer time of drug release through the skin. In conclusion, niosomes and liposomes are promising carriers for dermal delivery of the antioxidant extract Annona squamosa.
\end{abstract}

Keywords: Annona squamosa. Dermal delivery. Liposomes. Niosomes.

\section{INTRODUCTION}

Plants are considered as a source of secondary metabolites (Phytochemicals) like flavonoids, phenols, phenolic glycosides, saponins and glycosides with a variety of structural arrangements and properties. These secondary metabolites are highly biological active (De Fatima et al., 2006). Phytochemicals have protective or disease preventive properties including - antibacterial, anticancer, antifungal, and antioxidant that can protect the health (Krishnaraju, Rao, Sundararaju, 2005).

Since chemotherapeutics have not increasingly failed and antibiotic resistance exhibited by pathogenic microbial infectious agents has led to the screening of several medicinal plants for their potential antimicrobial activity (Colombo, Bosisio, 1996; Iwu, Duncan, Okunji, 1999).

Natural antimicrobials can be derived from seeds, stems, leaves, flowers and fruits of plants or from microorganisms. Even some therapeutic benefits can be

\footnotetext{
*Correspondence: E. A. Mohamad. Biophysics Department, Faculty of Science, Cairo University, Postal address: 12613 - Giza, Egypt. Mobile number: +201015082266. E-mail: ibtsamag_m@yahoo.com
}

traced to specific plant compounds, many herbs contain dozens of active constituents that, together, combine to give the plant its therapeutic value.

Annona squamosa L. (A. squamosa), usually recognized as sugar apple, is a tree growing in dry climes. All parts of $A$. squamosa have been traditionally utilized in the handling of many diseases (Suresh et al., 2006). A. squamosa has been employed as anti-inflammatory agent (Kirtikar, Basu, 1987). The beneficial medicinal properties of A. squamosa refer mainly to its contents of phytosterols, phenolic compounds, tannins, flavonoids, coumarin glycosides and terpenoids (Shalini, Sampathkumar, 2012). The extracts of different solvents of A. squamosa showed antioxidant properties (Jagtap, Bapat, 2012). Acetogenins and phenol contents of $A$. squamosa ethyl acetate leaf extract have high antioxidant activity and so, can scavenge free radical effectively (Sarma et al., 2015; Surendra, Male, Ratala, 2016). Transdermal drug delivery has many advantages over other established routes of drug delivery (Tuan-Mahmood et al., 2013). It can offer a non-invasive alternative to parenteral routes, thus circumventing issues such as needle phobia (Han, Das, 2015). A great surface area of skin and ease of 
access allows many placement options on the skin for transdermal absorption (Schoellhammer, Blanlschtein, Langer, 2014). In addition, it can improve patient compliance due to the reduction of dosing frequencies and is likewise suited for patients who are unconscious or vomiting, or those who rely on self-administration (Prausnitz, Langer, 2008). Transdermal drug delivery avoids pre-systemic metabolism, and so, can improve bioavailability (Ita, 2014; Han, Das, 2015).

Transdermal drug delivery has made a significant contribution to medical practice, but didn't yet achieve its potential as an alternative to hypodermic injections and oral delivery. The penetration of therapeutic compounds into the skin is impeded by the impermeability of the outermost layer of the skin, named stratum corneum (Amjadi, Mostaghaci, Sitti, 2017). Liposomes are efficient for the transdermal drug delivery as they are capable to control the release of the therapeutic agent. In summation, they may offer a localized depot in the skin, which brings down the quantities of drug permeating through the skin, thus minimizing systemic effects. Further, liposome vesicles can enhance transdermal drug delivery, increasing systemic drug concentrations. Moreover, the use of liposomes in nanocosmetology also has many benefits, including improved penetration and dispersion of active constituents, transport of active components, extended discharge time, greater stability of dynamic components, reduction of unwanted side effects besides high biocompatibility (Siler-Marinkovic, 2016). It has likewise been demonstrated that in transdermal route of delivery, when the drug is incorporated in niosomes, the penetration of the drug through the skin is greatly enhanced (Muzzalupo, Tavano, 2015).

A. squamosa extract compositions are lipophilic, thus, it is insoluble in water, but is soluble in organic solvents; therefore, suitable carriers must be used to deliver it and improve its bioavailability through the skin. The present work aims to study the properties of two different carriers for $A$. squamosa extract: liposomes and niosomes, which are considered as popular carriers. The study extends also to evaluating the potential of the transdermal bioavailability and release of $A$. squamosa after being loaded in niosomes / liposomes.

\section{MATERIAL AND METHODS}

\section{Material}

Methanol was purchased from Fisher Scientific UK. Polyoxyethylene-80 (tween 80 ) (purity $\geq 99 \%$ ) was supplied from Bio Basic Canada Inc. Phosphate buffer saline (PBS) pH 7.4 at $25{ }^{\circ} \mathrm{C}$ (purity $\geq 99 \%$ ) was purchased from Bio Shop Canada Inc. Diethyl ether (purity $\geq 99.7 \%$ ), cholesterol (purity $\geq 99.7 \%$, M Wt. 386.65), 1,2-dipalmitoyl-Sn-glycero-3phosphocholine (DPPC) (purity $\geq 99 \%$ ), Rhodamine B (purity $\geq 95 \%$, M Wt. 479.01) and chloroform solution (HPLC grade) were purchased from Sigma Aldrich. All chemicals were used without any further purification.

\section{Methods}

\section{Preparation and Identification of Annona squamosa} leaves extract

Leaves of $A$. squamosa (Family: Annonaceae) were collected during December 2017 from the medicinal garden of Ghabor Farm, egypt. To clean them, plant leaves were washed very well with tap water. The leaves were left to dry in a hot air oven dried at $55{ }^{\circ} \mathrm{C}$ (Ashok et al., 2009; Kashyap et al., 2015), and then, they were crushed into powder using an electric blender. The powder was then stored in plastic bags until use.

An amount of $6 \mathrm{~g}$ of the powdered A. squamosa. leaves were extracted using $20 \mathrm{ml}$ of methanol: distilled water (8:2), the extract was then centrifuged at $3000 \mathrm{rpm}$ for $15 \mathrm{~min}$, then, the supernatant was collected and filtered using Whatman paper No. 1. Ultimately, the solvents were allowed to be evaporated and the extract was concentrated using a rotary evaporator ( RV05ST, Germini BV laboratory) at $45^{\circ} \mathrm{C}$. The extract was analyzed using gas chromatography-mass spectroscopy system (SHIMADZU QP2010, Japan) and High Performance Liquid Chromatography (HPLC) system (Camag, Muttenz, Switzerland) to detect the main components of $A$. squamosa extract. The remaining extract was kept in the refrigerator until being used. The extraction yield was dependent on the initially used powder (El-Chaghaby, Ahmad, Ramis, 2011). After the botanical identification, the exsicata was deposited in Faculty of Science, Cairo University herbarium under the number 1272 .

\section{Preparation of niosomes entrapping A. squamosa leaves extract}

Niosomes were prepared by the thin film hydration method. $10 \mu \mathrm{g}$ Tween 80 and $3 \mathrm{mg}$ cholesterol in the ratio $(2: 1)$ were dissolved in $10 \mathrm{ml}$ ethanol in a round bottom flask with $1 \mathrm{ml}$ of A. squamosa extract.

The ethanol was then evaporated at $45^{\circ} \mathrm{C}$ under reduced pressure using a rotary evaporator at $50 \mathrm{rpm}$ producing a dry thin film. The hydration of the thin film was then done using PBS (pH 7.4). The formed niosomes 
were then subjected to ultrasonic sonication ( $5 \mathrm{~min}$ ) that led to the formation of small vesicles. Finally, niosomes were precipitated using a high speed cooling centrifuge (VS-18000M, Korea, power $220 \mathrm{~V} / 50 \mathrm{~Hz})(10000 \mathrm{rpm} x$ 30 min) (Sathali, Rajalakshmi, 2010).

\section{Preparation of liposome entrapping A. squamosa leaves extract}

An amount of $5 \mathrm{mg}$ DPPC lipids was first dissolved and mixed with $5 \mathrm{ml}$ chloroform to ensure a homogeneous mixture of lipids with $1 \mathrm{ml}$ of $A$. squamosa extract. $5 \mathrm{ml}$ chloroform was then, removed by rotary evaporation to obtain a thin lipid film on the sides of a round bottom flask. The lipid film was thoroughly dried to remove residual organic solvent by placing the flask on a vacuum pump for almost 90 minutes. Hydration of the dry lipid film was achieved by adding an aqueous solution of $90 \%$ phosphate buffered saline (PBS) to the container of the dry lipid film and agitating at a temperature slightly above the phase transition temperature of the lipid $\left(50^{\circ} \mathrm{C}\right)$ (Torchilin, Weissing, 2003).

\section{Characterization of niosomes and liposomes}

- Entrapment efficiency

The capacity of niosomes and liposomes to entrap A. squamosa extract was determined by the centrifugation method (Bendas, Tadros, 2007). Briefly, samples were centrifuged at 12,000 rpm (VS-18000 M, Korea, power $220 \mathrm{~V} / 50 \mathrm{~Hz}$ ) for $30 \mathrm{~min}$, to separate the free drug (supernatant) from the encapsulated one (pellet). The clear supernatant was then collected and vortexed to obtain a homogeneous solution, while the pellets obtained after centrifugation were diluted with $10 \mathrm{ml}$ saline buffer ( $\mathrm{pH}$ 7.4) and sonicated for $10 \mathrm{~min}$ for further use. This process was repeated three times for each sample.

The absorbance of $A$. squamosa was measured at different concentrations using a UV-visible spectrophotometer (JENWAY 6405, U.K.) at $270 \mathrm{~nm}$ (the resonance absorption of $A$. squamosa). The calibration curve of $A$. squamosa was made by plotting the absorbance against the concentration. The absorbance of the free A. squamosa in the supernatant was determined spectrophotometrically at $270 \mathrm{~nm}$. The concentration of the free drug in the supernatant was calculated from the calibration curve made for A. squamosa and the encapsulation efficiency for noisome and liposome was calculated from the following equation:

Entrapment efficiency $(\%)=\frac{\text { Initial concentration }- \text { Final concentration }}{\text { Initial concentration }} \times 100$
- Study of the morphology using transmission electron microscope (TEM)

Niosomes and liposomes were analyzed using a JEOL JEM.1230, Japan. The TEM microscope was operating at an accelerating voltage of $100 \mathrm{kV}$. Niosome and liposome suspension-entrapping extracts were negatively stained with a $1 \%$ aqueous solution of phosphotungstic acid, left to air-dry about $1 \mathrm{sec}$ then samples were incubated for approximately 10 minutes on perforated carbon-coated grids, and then analyzed.

- Study of the surface morphology using scanning electron microscope (SEM)

Niosomes and liposomes were freeze-dried and were coated with platinum for $5 \mathrm{~min}$. The surface morphology of the samples was visualized by SEM (Model Quanta 250 FEG-Field Emission Gun), with accelerating voltage of $30 \mathrm{KV}$

- Fourier transform infrared spectroscopy (FTIR)

FTIR is a helpful technique used to distinguish the types of functional groups and chemical bonds found in compounds. Freeze-dried powder of liposome and niosome were tested using NICOLET 6700 FT-IR Thermo scientific spectrometer, England, at a resolution of $4 \mathrm{~cm}^{-1}$ with a scan range from 400 to $4000 \mathrm{~cm}^{-1} .10 \mathrm{mg}$ of the dried extract powder was mixed with potassium bromide $(\mathrm{KBr})$ pellet for FTIR investigation.

\section{- In vitro extract release}

The in vitro release of $A$. squamosa extract from niosome and liposome was assayed using the dialysis technique in phosphate buffer solution (PBS, pH 7.4) for separating the release extract from niosome and liposome (Trotta et al., 2002; Foco, Gašperlin, Kristl, 2005; Maestrelli et al., 2005). Briefly, $3 \mathrm{ml}$ of niosome or liposome entrapped extract suspension was put into a cellulose acetate dialysis bag (Spectra/ Por, MW cutoff 12,000, Spectrum, Canada) immersed in $100 \mathrm{ml}$ of PBS and magnetically stirred at $50 \mathrm{rpm}$ (Model TK22, Kartell Italy). $2 \mathrm{ml}$ of the immersing solution was taken at different time intervals (every $1 \mathrm{~h}$ ), with replacing with equal volumes of fresh PBS. The absorbance of the samples was measured using at $270 \mathrm{~nm}$ using UNICO UV-2000 spectrophotometer, China. When the concentration of the extract in the receiving medium became constant, the experiment was stopped.

\section{Cellular uptake of niosomes and liposomes}

Penetration experiment was carried out using adult albino male rats weighing $\sim 120 \mathrm{~g}$. Rats were 
sacrificed by sudden decapitation. The abdominal skin region was carefully removed using fine scissor and forceps. Skin was taken out and then, the fatty material was removed and the skin was freshly used (Nayak, Mohanty, Sen, 2010). All animal experiments were performed in accordance with the Guidelines for Ethical and Regulatory for Animal Experiments as defined by the Cairo University, Egypt.

The uptake and distribution of niosomes and liposomes in the rat skin was visualized by confocal microscope using Rhodamine B dye. Briefly, $1 \mathrm{~mL}$ of niosome / liposome was mixed with Rodamine B ( $1 \mathrm{mg} / 5 \mathrm{~mL}$ of PBS). Rhodamine B-labeled niosome and liposome solutions were incubated on the skin for $2 \mathrm{~h}$ at $37^{\circ} \mathrm{C}$, then, the skin was sectioned with forceps. The skin sections were observed at emission wavelength of $545 \mathrm{~nm}$ by confocal laser scanning microscope (CLSM), Device Model: LSM 710, Software version: ZEN 2009, (Carl Zeiss, Jena, Germany).

\section{RESULTS AND DISCUSSION}

\section{Identification of $A$. squamosa main components}

Gas chromatography mass spectroscopy (GC/MS) was used to analyze the polar volatile compounds. Eight volatile compounds were identified in methanol fraction of A. squamosa leaf extract. Retention Time (RT), area of the peak, height, and peak width at half maximum (W05) were presented in Table I and its corresponding chromatogram (Figure 1).

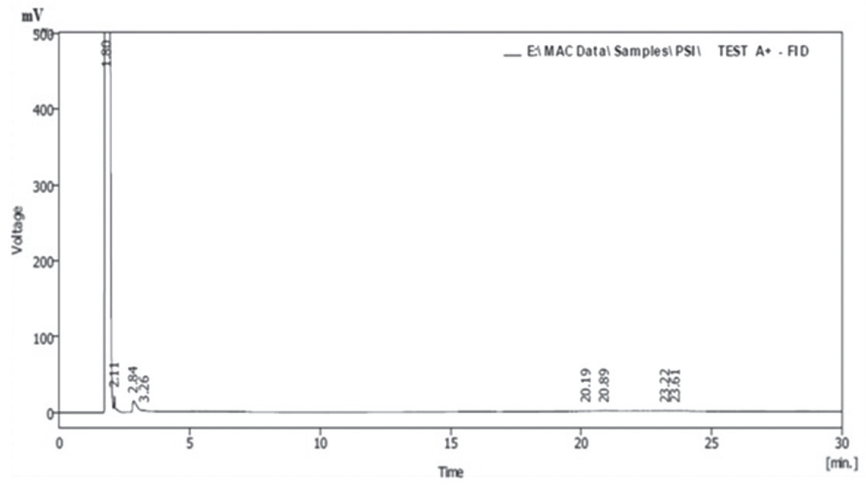

FIGURE 1 - GC/MS Chromatogram obtained for the methanol fraction of $A$. squamosa leaf extract.

\section{Entrapment efficiency}

In the present study, liposome exhibits higher efficiency for entrapping $A$. squamosa extract (67 $\pm 3 \%$ ) when compared to that of niosome $(24 \pm 3 \%)$. Encapsulation was optimized by investigating various processes and formulations.

\section{Morphology and characterization}

\section{SEM surface morphology}

As seen in Figure 2, the scanning electron microscope (SEM) images of $A$. squamosa extract-entrapped niosome and liposome were smooth having spherical surfaces with no pores. Particle size ranged from 160 up to $300 \mathrm{~nm}$ in the case of niosome and from 300 up to $500 \mathrm{~nm}$ in the case of liposome.

TABLE I - GC/MS analysis shows the main volatile components for the methanol fraction of $A$. squamosa leaf extract

\begin{tabular}{|c|c|c|c|c|c|c|c|}
\hline \multicolumn{8}{|c|}{ Results Table (Uncal - E:|MAC DatalSamples\PSI) TEST A+ - FD) } \\
\hline Peak & $\begin{array}{c}\text { RT } \\
{[\mathrm{min}]}\end{array}$ & $\begin{array}{c}\text { Area } \\
{[\mathrm{mV} . \mathrm{s}]}\end{array}$ & $\begin{array}{c}\text { Height } \\
{[\mathrm{mV}]}\end{array}$ & $\begin{array}{c}\text { Area } \\
{[\%]}\end{array}$ & $\begin{array}{c}\text { Height } \\
{[\%]}\end{array}$ & $\begin{array}{c}\text { W05 } \\
{[\mathrm{min}]}\end{array}$ & Compound Name \\
\hline 1 & 1.8 & 110395.3 & 12423.546 & 99.7 & 99.7 & 0.16 & Solvent (methanol) \\
\hline 2 & 2.11 & 82.864 & 21.746 & 0.1 & 0.2 & 0.04 & Limonene \\
\hline 3 & 2.84 & 168.324 & 14.521 & 0.2 & 0.1 & 0.18 & $R$-Carvone \\
\hline 4 & 3.26 & 12.954 & 1.474 & 0 & 0 & 0.14 & Spermidine \\
\hline 5 & 20.19 & 3.141 & 0.248 & 0 & 0 & 0.1 & $\begin{array}{c}\text { 5-Methyl } \\
\text { 1-5-(4,8,12,trimethytridencyl) } \\
\text { dihydro-2 }(3 H) \text {-furanone }\end{array}$ \\
\hline 6 & 20.89 & 12.257 & 0.458 & 0 & 0 & 0.28 & 16-Hentriacontanone \\
\hline 7 & 23.223 & 5.937 & 0.435 & 0 & 0 & 0.15 & Adenosine 3 phosphoric acid \\
\hline \multirow[t]{2}{*}{8} & 23.61 & 9.586 & 0.421 & 0 & 0 & 0.14 & Midacamide \\
\hline & Total & 110690.36 & 12462.848 & 100 & 100 & & \\
\hline
\end{tabular}




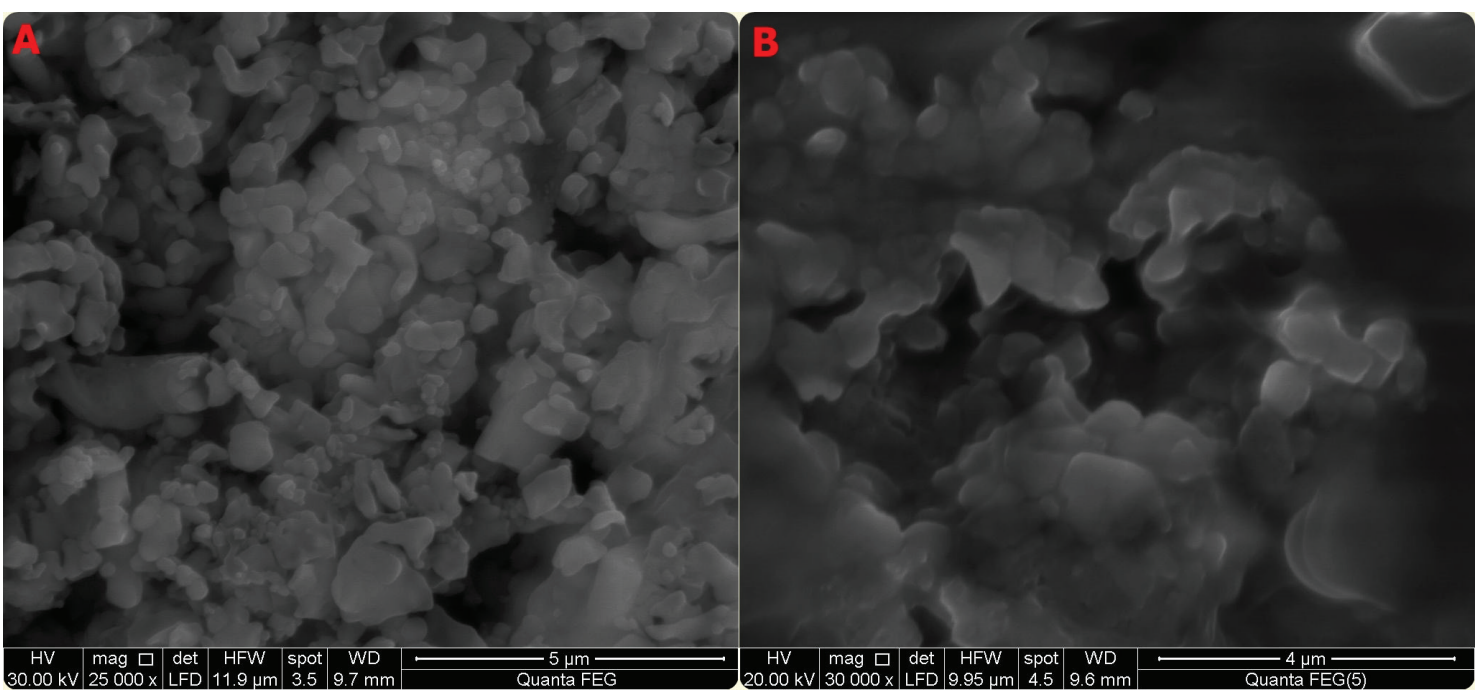

FIGURE 2 - The scanning electron microscope (SEM) images of A) A. squamosa extract-entrapped liposome and B) A. squamosa extract-entrapped niosome.

\section{Transmission electron microscope}

The examination of the ultrastructure using TEM revealed that the prepared $A$. squamosa-entrapped niosomes/liposomes were non-aggregated spherical shape particles (Figure 3), with sizes in agreement with those obtained by SEM. The morphology of each image indicated the homogeneity of the particle size as SEM focuses on the sample's surface and its composition whereas TEM provides the details about internal composition. Niosome and liposome suspensionentrapping extracts were negatively stained with a $1 \%$ aqueous solution of phosphotungstic acid. Samples were incubated for about 10 minutes on perforated carboncoated grids, and then examined.

Figure 3a shows blank niosomes having sphericalshaped particles with size slightly lower than the two other formulations, niosomes entrapping $A$. squamosa and liposomes entrapping A. squamosa, the entrapping drug in nanocarrier is larger than the blanks (Carugo et al., 2016).

All formulations exhibited a more narrow size distribution. The electrostatic charge repulsion between the similar charge (either positive or negative) particles prevents the aggregation and thus ensure a dispersed state of the nanosuspension in all the three formulations. The agglomerates in Figure $3 \mathrm{c}$ are not aggregated, it just a closely behind particles.

\section{FTIR}

FTIR spectrum (Figure 4) demonstrates the successful entrapment of $A$. squamosa into niosomes and liposomes. Upon adding $A$. squamosa extract in liposome / niosome, their FTIR spectra didn't change, which means that no additional chemical reactions took place. $A$.

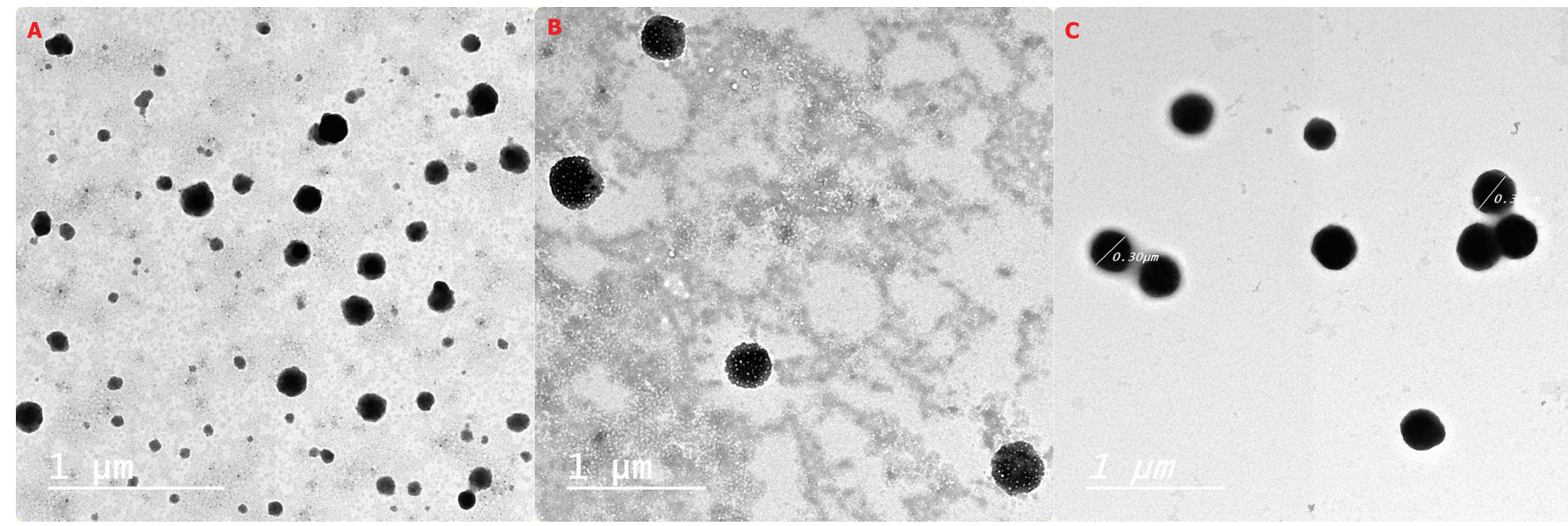

FIGURE 3 - Transmission electron microscope (TEM) images of A) Blank noisome; B) A. squamosa extract-entrapped noisome and C) A. squamosa extract-entrapped liposome. 

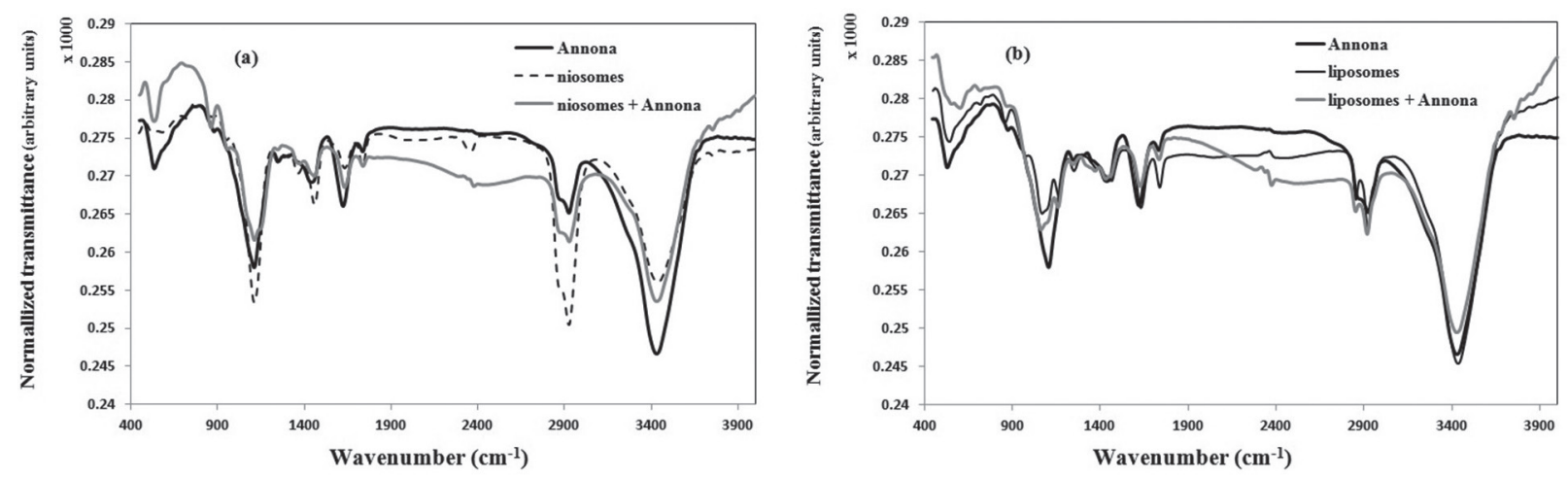

FIGURE 4 - FTIR spectra demonstrating the successful entrapment of $A$. squamosa into a) niosomes and b) liposomes.

squamosa was entrapped in the niosomes and liposomes bilayer membrane due to its hydrophobicity.

FTIR spectra of all samples showed characteristic bands of $\mathrm{CH}_{2}\left(2855 \mathrm{~cm}^{-1}\right), \mathrm{CH}_{3}\left(2925 \mathrm{~cm}^{-1}\right), \mathrm{C}-\mathrm{O}$ (around $1750 \mathrm{~cm}^{-1}$ ), C-O-C (around $1124 \mathrm{~cm}^{-1}$ ), Alkenyl $\mathrm{C}=\mathrm{C}$ stretch $\left(1644 \mathrm{~cm}^{-1}\right)$, Hydroxyl group in addition to $\mathrm{H}$-bonded $\mathrm{OH}$ stretch $\left(3210-3650 \mathrm{~cm}^{-1}\right.$ broad) (Coates, 2016).

\section{In vitro A. squamosa extract released from niosomes} and liposomes

The release of $A$. squamosa from the dialysis bag was in burst mode and reached equilibrium within $4 \mathrm{~h}$ (Figure 5), since its molecules were unrestricted and have free movement as the accumulative percentage release was estimated. For A. squamosa-entrapped niosomes / liposomes, since A. squamosa was located within the niosome and liposome membranes, release from niosomes and liposomes takes longer time interval. The present formulations have been designed to prolonge the release of A. squamosa as nano- encapsulation protects the molecules from premature degradation, improves their solubility, and promotes controlled drug release (Akl et al., 2016).

Figure 5 shows a sustained release of $A$. squamosa from niosome / liposome within $12 \mathrm{~h}$ in PBS buffer, room temperature and $\mathrm{pH}$ 7.4. A. squamosa released steadily and slowly over the time of the experiment which means that niosomes and liposomes are stable carriers for the sustained release of $A$. squamosa and this may enhance to save the therapeutic effects of $A$. squamosa. Liposome and niosome leading to a higher diffusion resistance compared to diffusion resistance seen in A. squamosa alone, which promotes their prolonged release. Values of the diffusion resistance in liposome and niosome dispersions are higher of those associated with unencapsulated $A$. squamosa. This implies that liposomes and niosomes are promising vehicles for protection and sustained release of A. squamosa (Pravilovic et al., 2015).

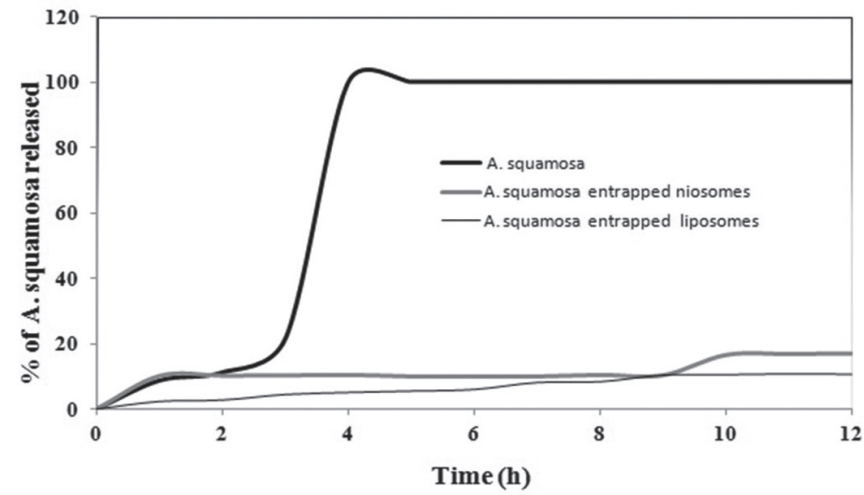

FIGURE 5 - In vitro release curves of $A$. squamosa from noisome and lisposome.

\section{Cellular uptake of niosomes and liposomes using ex-vivo skin tissue}

Rhodamine-labeled niosome / liposome nanoparticles were applied onto the rat skin tissue to test the penetration of both formulations across the different skin layers. In Figure 6, Rhodamine B-labelled niosome/liposome penetrate the stratum corneum and deposit between epidermis/dermis in $2 \mathrm{~h}$. These results suggested that niosmes and liposomes could be used as promising skin delivery carriers for $A$. squamosal, in addition, they led to the deposition of $A$. squamosa extract in epidermis and dermis, which leads to save the skin from accumulated oxidants by enhancing the hydrophilicity of the extract. These carriers were able to transmit $A$. squamosa extract to the skin by reasonable concentrations as the efficiency of liposomes entrapping $A$. squamosa is $67 \pm \%$ and the intensity of penetrated labeled liposomes was high (Figure 6b). Whereas liposome leads to a higher diffusion resistance that provides long periods of release. As shown in Figure 6a\&b, A. squamosa-entrapped liposome penetrated skin slightly deeper than squamosaentrapped niosomes and the penetration of both carriers was significantly higher than the penetration of Rhodamine only (Figure 6c). 


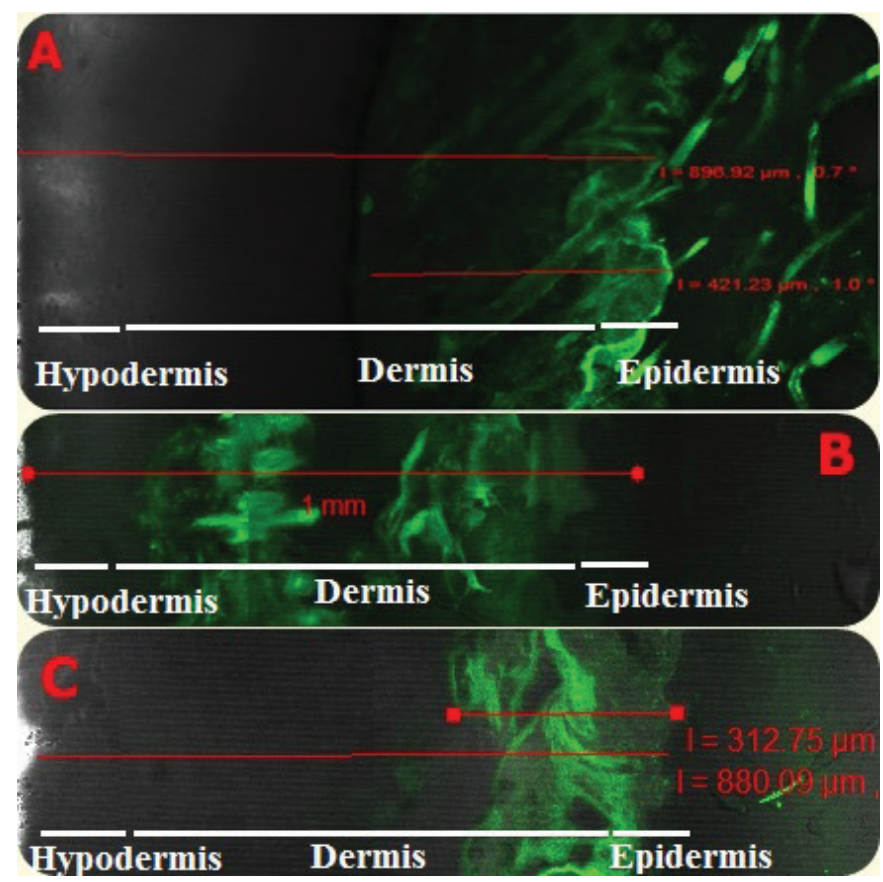

FIGURE 6 - Confocal microscope images of the penetration of A) A. squamosa extract-entrapped noisome; B) A. squamosa extract-entrapped liposome and C) Free Rhodamine across the different skin layers.

\section{CONCLUSION}

Niosomes and liposomes act as biological carriers by entrapping $A$. squamosa in their membrane, at the same time save its structure. Since $A$. squamosa extract rapidly oxidizes like most natural plant extracts and its structures break through the digestive system. These carriers significantly decrease the burst release of the extract as they have high extract diffusion resistance.

Additionally, niosomes and liposomes can be deposited in the epidermis and dermis, with more intense dermal distribution of A. squamosa in the case of liposome carriers and therefore could lead to antioxidant effect to the skin over a controlled release period. The two carriers penetrate skin in significant depth and this recommends the external use of $A$. squamosa entrapped niosomes and liposomes to scavenge skin from accumulative oxidants.

\section{CONFLICT OF INTEREST}

The authors declare no conflict of interest.

\section{FUNDING}

This research did not receive any specific grant from funding agencies in the public, commercial, or not-forprofit sectors.

\section{REFERENCES}

Akl MA, Kartal-Hodzic A, Oksanen T, Ismael HR, Afouna MM, Yliperttula M, Viitala T. Factorial design formulation optimization and in vitro characterization of curcumin-loaded PLGA nanoparticles for colon delivery. J Drug Deliv Sci Technol. 2016;32:10-20.

Amjadi M, Mostaghaci B, Sitti M. Recent advances in skin penetration enhancers for transdermal gene and drug delivery. Curr Gene Ther. 2017;17(2):139-146.

Ashok AV, Srinivas A, Ravitej K, Gopinadh V. Ethylacetate extract of annona squamosa seeds containing anti-head lice activity. Pharmacog J. 2009;1(3):207-209.

Bendas R, Tadros I. Enhanced transdermal delivery of Salbutamol sulfate via ethosomes. AAPS Pharm Sci Tech. 2007;8(4):213-220.

Carugo D, Bott E, Owen J, Stride E, Nastruzzi C. Liposome production by microfluidics: potential and limiting factors. Scient Rep. 2016;6:25876.

Coates J. Interpretation of infrared spectra, a practical approach. In: Encyclopedia of Analytical Chemistry. Wiley; 2016. p.1-23.

Colombo ML, Bosisio E. Pharmacological activities of Chelidoniummajus L. (Papaveraceae). Pharmacol Res. 1996;33(2):127-134.

De Fátima A, Modolo V, Conegero S, Pilli A, Ferreira V, Kohn K, De Carvalho E. Styryl lactones and their derivatives: biological activities, mechanisms of action and potential leads for drug design. Curr Med Chem. 2006;13(28):3371-3384.

El-Chaghaby A, Ahmad F, Ramis S. Evaluation of the antioxidant and antibacterial properties of various solvent extracts of Annona squamosal leaves. Arabian J Chem. 2011;7(2):227-233.

Fočo A, Gašperlin M, Kristl J. Investigation of liposomes as carriers of sodium ascorbyl phosphate for cutaneous photoprotection. Int J Pharm. 2005;291(1-2):21-29.

Han T, Das DB. Potential of combined ultrasound and microneedles for enhanced transdermal drug permeation: a review. Eur J Pharm Biopharm. 2015;89:312-328.

Ita K. Transdermal drug delivery: progress and challenges. J Drug Deliv Sci Technol. 2014;24(3):245-250. 
Iwu MW, Duncan AR, Okunji CO. New antimicrobials of plant origin. In: Janick J (editor). Perspectives on New Crops and New Uses. Alexandria, VA: ASHS Press; 1999; 457-462.

Jagtap B, Bapat A. Antioxidant activities of various solvent extracts of custard apple (Annona squamosa L.) fruit pulp. Nutrafoods. 2012;11(4):137-144.

Kashyap D, Sarmah P, Sultana S, Hub IB. Evaluation of antioxidant activity and nutraceutical property of annona squamosa L. fruits found in Brahmaputra valley agro-climatic. WJPPS. 2015;4(5):1151-1157.

Kirtikar R, Basu D. Indian Medicinal Plants. Dehradun: International Book Distributors; 1987; I-IV.

Krishnaraju AV, Rao TVN, Sundararaju D. Assessment of bioactivity of Indian medicinal plants using Brine shrimp (Artemiasalina) lethality assay. Int J Appl Sci Eng. 2005;3(2):125-134.

Maestrelli F, González-Rodríguez M L, Rabasco M, Mura P. Preparation and characterisation of liposomes encapsulating ketoprofen-cyclodextrin complexes for transdermal drug delivery. Int J Pharm. 2005;298(1):55-67.

Muzzalupo R, Tavano L. Niosomal drug delivery for transdermal targeting: recent advances. Res Rep Transdermal Drug Deliv. 2015;4:23-33.

Nayak K, Mohanty B, Sen K. Comparative evaluation of in vitro diclofenac sodium permeability across excised mouse skin from different common pharmaceutical vehicles. Int J Pharm Tech Research. 2010;2(1):920-930.

Prausnitz MR, Langer R. Transdermal drug delivery. Nat Biotechnol. 2008;26(11):1261-1268.

Pravilovic R, Radunovic V, Boskovic-Vragolovic N, Bugarski $\mathrm{B}$, Pjanovic R. The influence of membrane composition on the release of polyphenols from liposomes. Hemijska Industrija. 2015;69(4);347-353.

Sarma A, Kashyap D, Sarmah P, Sultana S, Hub B. Evaluation of antioxidant activity and nutraceutical property of annona squamosa L. Fruits found in Brahmaputra Valley agro-climatic condition. World J Pharm Pharm Sci. 2015;4(5):1151-1157.
Sathali H, Rajalakshmi G. Evaluation of transdermal targeted niosomal drug delivery of terbinafine hydrochloride. Int J Pharm Tech Res. 2010;2(3):2081-2089.

Schoellhammer CM, Blankschtein D, Langer R. Skin permeabilization for transdermal drug delivery: recent advances and future prospects. Expert Opin Drug Deliv. 2014;11(3):393407.

Shalini S, Sampathkumar P. Phytochemical screening and antimicrobial activity of plant extracts for disease management. Int J Curr Sci. 2012;209-218.

Siler-Marinkovic S. Liposomes as drug delivery systems in dermal and transdermal drug delivery. In: Dragicevic N, Maibach H (editors). Percutaneous penetration enhancers chemical methods in penetration enhancement. Berlin, Heidelberg: Springer; 2016.

Surendra G, Male A, Ratala R. DPPH Free radical scavenging activity of Annona squamosa Linn leaf extracts. IOSR-JPBS. 2016;11(5):4-6.

Suresh K, Mamoharan S, Panjamurthy K, Kavitak K. Chemopreventive and antilipidperoxidative efficiency of Annona squamosa bark extract. Pakistan J Biol Sci. 2006;9(14):2600-2605.

Torchilin P, Weissing V. Liposomes Practical approach. Oxford: Oxford Univ Press; 2003.

Trotta M, Peira E, Debernardi F, Gallarate M. Elastic liposomes for skin delivery of dipotassiumglycyrrhizinate. Int J Pharm. 2002;241(2):319-327.

Tuan-Mahmood T, McCrudden MT, Torrisi BM, McAlister E, Garland MJ, Singh TRR, Donnelly RF. Microneedles for intradermal and transdermal drug delivery. Eur J Pharm Sci. 2013;50(5):623-637.

Received for publication on $08^{\text {th }}$ February 2018 Accepted for publication on $23^{\text {rd }}$ October 2018 